\title{
Génétique formelle des pigmentations humaines à variations continues : beaucoup d'hypothèses, peu de conclusions
}

\author{
Catherine BONAITI et A. LANGANEY \\ U. 155, I.N.S.E.R.M., Château de Longchamps, 75016 Paris \\ Laboratoire d'Anthropologie, Musée de l'Homme, 75116 Paris
}

Résumé

Beaucoup de données ont été examinées dans le monde entier concernant la couleur de la peau et du poil, la possible adaptation, la physiologie, ainsi que d'éventuelles mutations suppresseuses. Dans la plupart des cas le caractère à étudier pour cette approche génétique est assez difficile à définir.

La génétique formelle de la variation quantitative de la couleur de la peau a été envisagée ici de deux manières:

1) avec des modèles mendéliens de 2 à 4 loci qui donnent en général un assez mauvais ajustement ;

2) avec des modèles quantitatifs qui débouchent sur un nombre de loci impliqués variant de 4 à 6 , ce qui est contraire à l'hypothèse de départ.

Dans ces conditions, il ne serait pas raisonnable d'accepter un modèle particulier malgré le fait qu'il y a bien un contrôle génétique de la couleur de la peau et du poil.

Beaucoup de connaissances et de mesures sont actuellement disponibles concernant la biochimie des pigments humains, leur génétique physiologique, les répartitions mondiales des pigmentations de la peau et des cheveux. La génétique formelle des déficiences en enzymes des chaînes de biosynthèse est aussi connue. Des relations indiscutables sont établies entre l'environnement et les pigmentations. Toutefois ces corrélations restent au niveau de l'évidence statistique et aucune des hypothèses, souvent très ingénieuses, qui ont été proposées pour les expliquer par des modèles sélectifs, n'a encore fait l'objet d'une démonstration véritable. Toute assertion dans ce domaine doit donc faire l'objet de réserves clairement exprimées.

La pigmentation de la peau ou des phanères, hormis les cas de mutations supprimant le pigment, est affaire de quantité et non de présence ou absence. L'objet de la mesure doit être clairement défini et répétable pour permettre la comparaison d'un individu à l'autre ou d'une population à l'autre. Dans le cas de la pigmentation des cheveux, l'intensité de la pigmentation varie considérablement avec 
l'âge, rendant difficiles l'interprétation de la plupart des enquêtes anthropologiques disponibles (Meyer-KöNIG, 1977 ; Reuer \& SEIDler, 1978). Dans le cas de la pigmentation de la peau, sa mesure varie beaucoup avec les parties du corps et leur éventuelle exposition au soleil. La facilité et la tradition veulent que l'on utilise désormais la spectrophotométrie, à une longueur d'onde de $685 \mathrm{~m}$ lı, et en un point bien précis du corps, aussi peu exposé que possible aux influences environnementales diverses (WEINER, 1951 ; HARRISON \& OWEN, 1956, 1957 ; BARNICOT, 1958 ; Leguebe, 1976). Toutefois, il est bien évident que la réalisation du caractère, au niveau microscopique, détient la clé des mécanismes génétiques. Celle-ci est malheureusement peu accessible et les rares enquêtes (SzABO, 1967) qui ont cherché à mettre en évidence des différences raciales dans les comptages de cellules et l'étude des formes et dimensions des mélanosomes sont trop partielles et peu significatives pour beaucoup nous avancer. Il semble, néanmoins, que la densité de mélanosomes épidermiques - toutes cellules comprises - soit l'une des variables les plus periinentes dans cette étude, et aussi, malheureusement, la plus délicate à mesurer.

Une fois soulignée la difficulté de définir le caractère intensité de pigmentation, il reste à étudier les modèles de transmission qui ont été proposés. Nous nous limiterons ici à la pigmentation de la peau, celles de l'œil ou des cheveux posant des problèmes spécifiques du fait d'une discontinuité partielle dans le premier cas et des variations avec l'âge dans le second. Il n'est pas inutile de rappeler que la génétique des caractères à variation continue reste au niveau de l'analyse statistique et se relie mal aux données de la génétique moléculaire.

Les premières études sont dues à G.C. et C.B. Davenport $(1910,1913)$ qui ont étudié les croisements entre Noirs et Blancs aux Bermudes et à la Jamaïque. Par comparaisons avec une toupie colorée dont la surface relative du secteur noir variait de 0 à 78 p. 100 , ils ont séparé les différents types de pigmentation en 5 classes. Les hybrides $F_{1}$ présentaient un degré de pigmentation intermédiaire entre Noirs et Blancs et les individus $F_{2}$ (issus d'un croisement $F_{1} \times F_{1}$ ) se répartissaient dans les 5 classes de pigmentation avec un maximum dans la classe 2 . Les auteurs concluaient que les données étaient compatibles avec l'hypothèse de deux locus indépendants comportant chacun des allèles ayant des effets additifs, chacun des locus ayant un effet équivalent sur le degré de pigmentation. Ceux-ci devaient néanmoins faire l'hypothèse que d'autres facteurs génétiques pouvaient avoir un rôle puisque les individus $F_{1}$ ne se trouvaient pas tous dans la classe 2, mais qu'un nombre non négligeable d'entre eux ( 24 p. 100) se trouvaient dans les classes 1 et 3 .

La principale critique que l'on peut faire au modèle de DAvenPort est d'avoir choisi arbitrairement 5 classes pour un caractère dont la distribution est manifestement continue.

En 1953, Stern a abordé le problème en utilisant les données que Hershovitz, avait rassemblées en 1930 sur les Noirs américains.

En faisant les hypothèses suivantes :

- les gènes jouant un rôle dans la pigmentation ont des effets égaux et additifs compte tenu de la mesure utilisée (pourcentage de secteur noir sur une toupie colorée);

- l'échelle utilisée est liée linéairement au nombre de gènes ;

- il y a panmixie et équilibre génétique, STERN a calculé les distributions théoriques des fréquences avec des modèles à respectivement $2,3,4,5,6,7,10$ 
et 20 couples de gènes et comparé ces données théoriques aux résultats observés par Herskovitz : il a trouvé que les meilleures estimations étaient données par les modèles à 4,5 et 6 couples de gènes mais devait néanmoins admettre que la concordance était loin d'être totale entre ces modèles théoriques et les observations. Il suggérait alors que les hypothèse de bases n'étaient sans doute pas exactes. Nous ne le contredirons certes pas sur ce dernier point, d'autant que BARNEs (1929) avait bien montré qu'il existait une homogamie phénotypique positive partielle pour le degré de pigmentation chez les Noirs américains.

A la même époque (1953) GATES proposait à son tour un modèle en se référant à d'autres observations. L'originalité de son modèle résidait dans le fait que les 3 locus - qu'il supposait être impliqués - auraient des effets inégaux sur le degré de pigmentation.

En 1964, Harrison \& OWEN publiaient les résultats de leur étude sur une communauté de Liverpool où vivaient des Blancs britanniques et des Noirs d'Afrique de l'Ouest. Ils ont mesuré la moyenne et la variance du pourcentage de réflexion au spectrophomètre chez 5 types d'individus :

- les Blancs;

- les Noirs;

- les hybrides $F_{1}$ (Blancs $\times$ Noirs);

- les back-cross $\left(F_{1} \times\right.$ Blancs et $F_{1} \times$ Noirs $)$;

- la $F_{2}\left(F_{1} \times F_{1}\right)$.

Ainsi que l'on pouvait s'y attendre dans le cas d'un caractère quantitatif, au moins en partie génétiquement déterminé, la moyenne pour la $F_{1}$ était intermédiaire entre celle des Blancs et celle des Noirs et les moyennes des back-cross se trouvaient entre ces dernières et celle de la $F_{1}$. En supposant un modèle multifactoriel (existence de nombreux gènes ayant des effets égaux et de facteurs environnementaux) et en utilisant les techniques de la génétique quantitative, ils ont conclu que la variance génétique additive était du même ordre de grandeur que la variance de l'environnement et que la variance de dominance était très faible. Ils ont également estimé que le nombre de locus dans la pigmentation de la peau était compris entre 3 et 4 . Cette estimation semble contradictoire avec l'hypothèse de base (grand nombre de locus) et aurait dû amener les auteurs, sinon à rejeter leurs résultats, au moins à mettre en doute la validité de l'analyse.

A la lumière de ces résultats, STERN (1970) reprenait ses modèles théoriques et se félicitait de trouver que les modèles qui s'accordaient le mieux avec les données étaient ceux où 3 ou 4 locus sont impliqués, et que cette fois l'écart entre la théorie et les observations était beaucoup moins important que dans son étude de 1953.

Dans l'ensemble, la difficulté de ce genre d'étude, lorsque les observations arrivent à rejeter un modèle mendelien simple, est que la discrimination entre toutes les combinaisons possibles selon le nombre de locus, le nombre d'allèles à chaque locus, l'importance des interactions entre allèles au niveau d'un locus et entre locus, et l'existence de facteurs d'environnement, devient impossible, les tests ne permettant de discriminer qu'entre des modèles extrêmes. Cotrerman (1953) avait montré que pour un seul locus, le nombre de phénogrammes augmentait considérablement selon que 2, 3 ou 4 allèles ségrégeaient à ce locus. La tentation est grande pour 
les auteurs de compliquer leur modèle en ajoutant des paramètres supplémentaires jusqu'à ce que celui-ci soit conforme aux observations. Cela ne prouve en aucun cas que ce modèle est effectivement le bon, d'autant que l'analyse permettant la confrontation entre le modèle et les données doit toujours s'appuyer sur un nombre important d'hypothèses qu'il est généralement impossible de tester.

On ne peut donc pas dire que l'on dispose d'une hypothèse satisfaisante concernant l'hérédité de l'intensité des pigmentations à variations continues.

Reçu pour publication en mars 1981.

\begin{abstract}
Summary
Formal genetics of human pigmentation with continuous variation : many hypothesis, a few conclusions
\end{abstract}

Considerable data has been provided about skin or hair colour throughout the world, possible adaptations, physiology and some suppressor mutations. In most cases the character to study in a genetic approach is quite difficult to define.

Formal genetics of quantitative variation of skin colour has been considered from two points of view :

1) Mendelian models dealing with 2 to 4 loci generally give a bad fit.

2) Quantitative models estimate to 4 to 6 the number of involved loci - which is contradictory to their hypotheses.

It should be quite unreasonnable, in such condition to believe in any particular model, despite concording evidences of a genetic control of skin and hair colours.

\title{
Références bibliographiques
}

Barnes I., 1929. The inheritance of pigmentation in the American Negro. Hum. Biol., 1. 321-381.

Barnicot N.A., 1958. Reflectometry of the skin in Southern Nigerians and in some Mulattoes. Hum. Biol., 30, 150.

Cotterman C.W., 1953. Regular two allele and three allele phenotype systems. Am. J. Hum. Genet., 8 (5), 193-235.

Davenport G.C. and C.B., 1910. Heredity of skin pigmentation in Man. Amer. Naturalist., 44, 641.

DaVenport C.B., 1913. Heredity of skin color in negro white crosses. Carnegie prest. Washington. Publ. 188.

HARRISON G.A. et OwEN J.J.T., 1956. The application of spectrophotometry to the study of skin colour inheritance. Acta Genet., 6, 481.

HARRISON G.A. et OWEN I.J.T., 1957. The measurement and inheritance of skin colour in Man. Eug. Rev., 49, 73-76.

Harrison G.A. et OwEN J.J.T., 1964. Studies on the inheritance of skin colour. Ann. Hum. Genet. Lond., 28, 27-37. 
Herskovitz M.J., 1930. The anthropometry of the American Negro. Coloumbia Univ. Contr. Anthrop., II, 1-283.

Leguebe A., 1976. Catalogue of spectrophotometric data on skin pigmentation. Institut royal des sciences naturelles de Belgique. Document 9, Bruxelles.

MeYer König L., 1977. Age specify change in the colour of hair and eyes. A longitudinal study. J. of Hum. Evol., 6, 711-713.

Reuer E. et Seidler H., 1978. Statistical progress in the change in hair colour from infancy to adult life. J. Hum. Evol., 7, 529-533.

STERN C., 1953. Model estimates of the frequency of white and near white segregants in the american Negro. Acta Genet., 4, 281.

Stern C., 1970. Model estimates of the number of gene pairs involved in pigmentation variability of the Negro American. Hum. Hered. (Basel), 20, 165-168.

Szabo, 1967. Phil. Trans. R. Soc. B, 252-447.

WeINER J.S., 1951. A spectrophotometer to measurement of skin colour. Man, 51, 253. 